\title{
Zur Kenntnis der Folgereaktionen
}

\author{
Nr. 4 \\ Das Konstantenverhältnis bei der sauren Verseifung \\ der Oxal- und Malonsäureester \\ von \\ Anton Skrabal und Danica Mrazek
}

Aus dem Chemischen Institut der k. k. Karl-Franzens-Universität zu Graz

(Vorgelegt in der Sitzung am 6. Juni 1918)

In der zweiten Mitteilung ${ }^{1}$ wurde die saure Verseifung des Oxalsäuremethylesters durch Bestimmung des Säuretiters messend verfolgt und gezeigt, daß für diesen Ester das Konstantenverhältnis $2: 1$ zurecht besteht, dab aber die einfachen Beziehungen durch Nebenvorgänge getrübt werden. Solche Yebenvorgänge sind die autokatalytische Beschleunigung der Reaktion durch die sich bildende Oxalsäure und Estersäure und die Bremsung der Reaktion gegen Ende durch die Gegenwirkung. Diese beiden Umstände lassen sich in ihrem Einfluß auf das Rechenergebnis vollkommen ausschalten, wenn $z w e i$ voneinander unabhängige Konzentrationen durch Analyse ermittelt werden, weil die beiden rariablen Konzentrationen und die aus ihnen berechneten Geschwindigkeitskonstanten durch die Nebenvorgänge in gleicher Weise beeinflußt werden. Die einwandfreie Feststellung des Verhältnisses der Geschwindigkeiten der beiden Verseifungsstufen ist aber gerade bei der Oxalsäure ron besonderem theoretischen Inter-

I A. Skrabal, Monatsh. f. Ch., 38 (1917), 29. 
esse, worauf in einer besonderen Arbeit zurückgekommen werden wird. Bei dieser Gelegenheit wurde auch die saure Verseifung des Malonsäureesters, über welche Reaktion im Schriftum kaum Angaben vorliegen, kinetisch gemessen.

Wir hatten anfangs die Absicht, als zweite variable Konzentration neben dem Säuretiter die der Oxalsäure nach der in der eben erwähnten Arbeit dargelegten Methode zu ermitteln. Bei der Durchführung der letzteren stießen wir auf Schwierigkeiten, die darin gelegen sind, daß das Calciumoxalat in der Kälte - wegen der weitergehenden Verseifung darf nicht erhitzt werden - - jn schwerfiltrierbarer, feinkörniger Form ausfällt, und daß es überdies in der zur Anwendung gebrachten Lösung von Ammonsalzen und Acetaten merklich löslich ist. Als analytisches Ergebnis unserer Versuche konnten wir feststellen, daß die Bestimmung des Kalks durch Fällung mit überschüssigem Oxalat, wie ja bekannt, keine wesentlichen Schwierigkeiten bereitet, daß aber die umgekebrte Fällung der Oxalsäure mit überschüssigem Calciumsalz mit Fehlern behaftet ist. Glücklicherweise wiesen uns die in der dritten Mitteilung ${ }^{1}$ gemachten Erfahrungen einen anderen Weg, der es ermöglichte, in dem gegebenen Reaktionsgemisch die Bestimmung des Neutralesters vorzunehmen. Versetzt man nämlich die neutralisierte Lösung von Mineralsäure, Neutralester, Estersäure und Oxalsäure mit Jodid-Jodat, so tritt eine langsame Jodausscheidung ein, die ihr praktisches Ende findet, wenn auf $1 \mathrm{Mol}$ Dimethyloxalat 1 Atom Jod ausgeschieden ist. Diese Methode fußt also darauf, daß der Neutralester entsprechend dem Konstantenverhältnis bei der alkalischen Verseifung in der Jodid-Jodatlösung sehr viel rascher (alkalisch) verseift als die Estersäure und gestattet es, den Neutralester auf jodometrischem Wege zu bestimmen.

Das Analysenverfahren bestand demnach darin, daß $100 \mathrm{~cm}^{3}$ des Reaktionsgemisches mit Ammoniak und Alizarin ${ }^{2}$

1. A. Skrabal, Monatsh. f. Ch., 38 (1917), 159.

2 A. Kailan, Zeitschr. f. physik. Chemie, 85 (1913), 706, hat für dieselbe Titration Rosolsäure benutzt. Wir haben auch diesen Indikator geprütt, aher den Alizarinumschlag schärfer befunden. 
als Indikator austitriert wurden (Alizarintiter $T_{a}$ ), worauf $4 \cdot 4 \mathrm{~g}$ Jodkalium und $10 \mathrm{~cm}^{3}$ einer Jodatlösung, enthaltend 0. $72 \mathrm{~g} \mathrm{KJO}_{3}$, hinzugesetzt und nach dreitägigem Stehenlassen im Dunkeln das gebildete Jod titriert (Jodtiter $T_{i}$ ) wurde. Diese Konzentrationen und die Wartezeit sind derart bemessen, daß nach dem Ergebnis der kinetischen Messungen in der dritten Mitteilung die Reaktion zwischen Neutralester und Jodid-Jodat so gut wie zu Ende sein muß.

Wir lassen nunmehr die Versuche folgen, bei deren Durchführung - und dasselbe gilt auch für die Versuche der folgenden Aibeiten -- mannigfache äußere Schwierigkeiten $z u$ überwinden und Störungen in den Kauf zu nehmen waren. Sie hatten ihre Ursache in der durch den Krieg bedingten, abwechselnd oder gleichzeitig herrschenden Not an Beheizungskohle, Leuchtgas, Léitungswasser und den erforderlichen Chemikalien, vor allem Methylalkohol, der immer wieder zurückgewonnen werden mußte, wenn unsere Arbeit nicht an Naterialmangel hätte scheitern sollen.

\section{Die Verseifung von Dimethyloxalat.}

Die Zusammensetzung des Reaktionsgemisches ist in Grammformelgewichten pro Liter, die Zeit $t$ in Minuten angegeben. Der azidimetrische Titer ist in Grammäquivalenten (Äqualen) angeführt. Aus ihm wurde die Konstante erster Ordnung $k_{a}$ berechnet, die für das Konstantenverhältnis $2: 1$ und bei von Störungen freiem Reaktionsverlauf konstant sein muB. Der Jodtiter ist entweder in Kubikzentimetern Thiosulfatlösung (ungefähr zehntelnormal) pro $100 \mathrm{~cm}^{\prime \prime}$ des Reaktionsgemisches angegeben oder auf Literäqualen umgerechnet $\left(T_{i}\right)$, in welchem Fall er die Konzentration des Neutralesters ausdrückt. Aus beiden Zahlenreihen berechnet sich dasselbe $k_{\|}$erster Ordnung, das ja von der Wahl der Konzentrationseinheit unabhängig ist, und das die Geschwindigkeit bedeutet, mit welcher der Neutralester nach der ersten Stufe verseift. Alle Messungen wurden bei $25^{\circ}$ angestellt. 
1. Versuch.

\begin{tabular}{|c|c|c|c|c|c|}
\hline 1 & $T_{i:}$ & am Thios. & $104 k_{i t t}$ & $10+k_{n}$ & $k_{a}$ \\
\hline 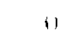 & $0 \cdot 1.10 \%$ & $92 \cdot 23$ & $\ldots$ & $\therefore$ & ... \\
\hline 130 & 11.1326 & $.2 \cdot 115$ & $9 \cdot 6$ & $39 \cdot 0$ & $2 \cdot 0$ \\
\hline $2+101$ & $0 \cdot 1431$ & $62 \cdot 53$ & $9 \cdot 3$ & 2010 & $2 \cdot 2$ \\
\hline 5ino & $0 \cdot 1837$ & $34 \cdot 45$ & $0 \cdot 9$ & $19 \cdot 6$ & $2 \cdot 0$ \\
\hline 640 & $0 \cdot 1982$ & $27 \cdot 31$ & $9 \cdot B$ & $16 \cdot 6$ & $1 \cdot \tau$ \\
\hline 1470 & $0 \cdot 2536$ & $6 \cdot 65$ & $9 \cdot 3$ & $16 \cdot 7$ & $1 \cdot 8$ \\
\hline 2004 & 0.2700 & $2 \cdot 37$ & $8 \cdot 2$ & $19 \cdot 3$ & $2 \cdot 3$ \\
\hline
\end{tabular}

Die beiden Koeffizienten $k_{u e}$ und $k_{t}$ werden durch die freiwerdende Säure stets und - wie in der folgenden Arbeit gezeigt werden wird - auch durch die Gegenwirkung dann in gleicher Weise beeinflußt, wenn auch für die Gegenreaktion das Konstantenverhältnis $2: 1$ zurecht besteht. Wenn also das erste der beiden Alkyle doppelt so rasch verseift als das zweite, so muß der Quotient aus $k_{n}$ und $k_{a}$ konstant gleich 2 sein. Tatsächlich schwankt dieser Quotient innerhalb der Versuchsfehler der Titrationen um den Wert 2 als Mittelwert.

Die mit Thiosulfat austitrierten Proben zeigten nach längerem Stehen Nachjodung, die nach einigen.'Tagen sebr beträchtlich war und offenbar auf die langsame Wechselwirkung zwischen Jodat und dem bei der Titration entstandenen Tetrathionat zurückzuführen ist.

\section{Versuch.}

\begin{tabular}{|c|c|c|c|c|}
\hline$t$ & $T_{4}$ & $T_{i}$ & $10^{4} h_{1 / 2}$ & $10 \pm k_{n}$ \\
\hline 0 & $0 \cdot 1047$ & 0.09872 & $\cdots$ & $\cdots-$ \\
\hline 60 & 1). 1146 & 0.08917 & $8 \cdot 7$ & $16 \cdot 9$ \\
\hline 1.54 & 1). 1286 & 0.07599 & $8 \cdot 4$ & $1.7 \cdot 0$ \\
\hline 314 & $0 \cdot 1550$ & 0.05905 & $8 \cdot 3$ & $15 \cdot 7$ \\
\hline 554 & (1). 1758 & $0 \cdot 04089$ & $7 \cdot 8$ & $15 \cdot 3$ \\
\hline 1394 & $0 \cdot 2315$ & 0.01240 & $6 \cdot 8$ & $14 \cdot 2$ \\
\hline 1954 & $0 \cdot 2484$ & 0.00629 & $5 \cdot 1$ & $12 \cdot 3$ \\
\hline 2884 & $0 \cdot 2602$ & 0) .00374 & $2 \cdot 8$ & $5: 6$ \\
\hline
\end{tabular}


Dieser Versuch zeigt den Einfluß eines Alkoholzusatzes. Er äußert sich nach zwei Richtungen: einmal darin, daß er nach dem Massenwirkungsgesetz die Gegenwirkung früher in Erscheinung treten läßt - das bewirkt ein stärkeres Abfallen der Konstanten gegen Ende der Reaktion - andrerseits verzögert der Alkohol die Verseifung, ${ }^{1}$ indem schon zu Beginn der Reaktion, wo die Gegenwirkung noch unmerklich ist, die Konstanten kleiner sind als in Versuch 1. Beide Einflüsse verändern $k_{u c}$ und $k_{n}$ in demselben Ausmaß und daher ist ibr Quotient abermals gleich 2.

Durch diese Versuche ist mit Sicherheit dargetan, daß im Oxalsäureester das erste Alkyl doppelt so rasch abgespalten wird als das zweite.

Eine etwaige Abweichung von diesem einfachen Konstantenverhältnis kann nur so gering sein, daß sie innerhalb der Fehler der benutzten Analysenmethode fällt.

\section{Die Verseifung von Diäthyloxalat.}

Die Bestimmung des Neutralesters mit Jodid-Jodat ist hier nicht durchführbar, weil dieser Ester, wie der eine von uns gezeigt hat,"- mit Trijodkalium eine schwerlösliche Doppelverbindung liefert. Es ließe sich zwar auf Grund dieser Tatsache eine andere Analysenmethode zur Bestimmung von Diäthyloxalat ausarbeiten und anwenden, doch haben wir hiervon Abstand genommen, weil das Konstantenverhältnis 2:1 für den Äthylester aus der Analogie mit dem Methylester. hervorgeht und sich überdies auch aus der annähernden Konstanz von $k_{u i}$ zur Genüge erweist. Der folgende Versuch, bei welchem der Reaktionsfortschritt aus dem Alizarintiter allein ermittelt wurde, wurde in der Absicht angestellt, die beiden Ester bezüglich ihrer Verseifungsgeschwindigkeit vergleichen zu können.

1 In den Virseifungskonstanten steckt die Wasserkonzentration. Da letztere durch den Mkoholiasatz verringert wird, mub die Verseifungskonstante kleiner werden. Nebenher kann auch Mediumkatalyse vorliegen.

2A. Skraba1, Ber. d. Deutschen ch. Ges, 50 (1917), 581. Eine Fortsetzung dieser Untersuchungen wird demnächst erscheinen. 
3. Versuch.

$\begin{array}{rcc}0 \cdot 1 \mathrm{HCl}+0 \cdot 1103 \mathrm{C}_{2} \mathrm{O}_{4}\left(\mathrm{C}_{2} \mathrm{H}_{5}\right)_{2} . \\ i & T_{a} & 10.4 k_{4 l e} \\ 0 & 0 \cdot 1143 & - \\ 61 & 0 \cdot 1208 & 5 \cdot 2 \\ 196 & 0 \cdot 1343 & 5 \cdot 2 \\ 457 & 0 \cdot 1615 & 6 \cdot 0 \\ 1788 & 0 \cdot 2568 & 6 \cdot 9 \\ 2872 & 0 \cdot 2906 & 6 \cdot 9 \\ 4353 & 0 \cdot 3077 & 5 \cdot 7\end{array}$

Der nach den Äquivalenten berechnete Koeffizient ist ungefähr konstant und läßt sehr deutlich die durch die freiwerdende Säure bedingte Beschleunigung der Verseifung erkennen, die erst gegen Schluß der Reaktion durch die Gegenwirkung ïberkompensiert wird. Vergleicht man die von Störungen freien Anfangswerte von Versuch 1 und 3 , so ergibt sich, daß der Methylester $9 \cdot 6: 5 \cdot 2=1 \cdot 8$-mal, also nicht ganz doppelt so rasch verseift als der Ähylester.

\section{Die Verseifung von Dimethylmalonat.}

Die Ermittlung des Säuretiters $T$ wurde mit Zehntelnormalbaryt und Phenolphtalein als Indikator vorgenommen. Weil der Malonester bei der Alkalinität, bei welcher dieser Indikator anspricht, schon ziemlich rasch nach der ersten Stufe alkalisch verseift, ist der Endpunkt der Titration zwar kurz, aber genügend anhaltend, um eine genaue Säuretitration $z$ li ermöglichen. Es ist nur durch langsames und unter Unschwenken durchzuführendes Zuflicßenlassen des Baryts dafür zu sorgen, daß in der zu titrierenden Lösung keine örtliche Anhüufung der Lauge stattindet. Nach den krfahrungen, die bei des sauren Verseifung des Kaliummethyloxalats gemacht wurden, war die Durchführbarkeit der Titration rorauszusehen, weil der Malonester nach der ersten und der Oxalester nach der zwreiten Stufe ungefähr gleich rasch alkalisch verseifen. 
Dic angewandten Ester waren Kahlbaum'sche Präparate, die durch gebrochene Destillation noch gereinigt wurden.

Bei den Versuchen wurden zu einer Analyse $25 \mathrm{~cm}^{3}$ des Reaktionsgemisches verwendet.

4. Versuch.

$0 \cdot 1 \mathrm{HCl}+0 \cdot 1001 \mathrm{CH}_{2}\left(\mathrm{CO}_{2} \mathrm{CH}_{3}\right)_{2}$.

\begin{tabular}{|c|c|c|c|}
\hline$l_{2}-t_{1}$ & $T$ & $(a-x-y) a c$ & $104 k_{a c}$ \\
\hline$\div$ & $0 \cdot 1027$ & 0.1975 & - \\
\hline 1396 & $0 \cdot 1301$ & $0 \cdot 1701$ & $1 \cdot 07$ \\
\hline 1494 & $0 \cdot 1546$ & $0 \cdot 1456$ & $1 \cdot 04$ \\
\hline 1330 & $0 \cdot 1738$ & $0 \cdot 1264$ & $1 \cdot 06$ \\
\hline 1755 & $0 \cdot 1952$ & $0 \cdot 1050$ & $1 \cdot 05$ \\
\hline 1130 & $0.207 t$ & $0 \cdot 0931$ & $1 \cdot 00$ \\
\hline 1420 & 0.2203 & $0 \cdot 0799$ & $1 \cdot 08$ \\
\hline 3243 & $0 \cdot 2434$ & $0 \cdot 0568$ & $1 \cdot 05$ \\
\hline 1433 & $0 \cdot 2512$ & $0 \cdot 0490$ & $1 \cdot 03$ \\
\hline- & 0.2592 & $0 \cdot 0410$ & - \\
\hline 2535 & $0 \cdot 268 \bar{z}$ & $0 \cdot 0317$ & $1 \cdot 02$ \\
\hline 3044 & $0 \cdot 2760$ & $0 \cdot 0242$ & $(0 \cdot 89)$ \\
\hline 4216 & (j) 2826 & $0 \cdot 0176$ & $(0 \cdot 76)$ \\
\hline \multirow[t]{2}{*}{4386} & $0 \cdot 2870$ & 0.0132 & $(0.65)$ \\
\hline & & & $1 \cdot 05$ \\
\hline
\end{tabular}

5. Versuch.

$0 \cdot 05 \mathrm{HCl}+0 \cdot 1000 \mathrm{CH}_{2}\left(\mathrm{CO}_{2} \mathrm{CH}_{3}\right)_{2}$.

\begin{tabular}{|c|c|c|c|}
\hline$t_{0},-t_{1}$ & $\%$ & $(a-x-y) a e$ & 11) $4 k_{\text {ble }}$ \\
\hline -. & $0 \cdot 0512$ & $0 \cdot 1988$ & ..- \\
\hline 1371 & $0 \cdot 0647$ & $0 \cdot 1851$ & 0.52. \\
\hline 1757 & 0.0825 & $0 \cdot 1675$ & $0 \cdot 57$ \\
\hline 1154 & 0.0909 & $0 \cdot 1591$ & $0 \cdot 45$ \\
\hline 1438 & $0 \cdot 1027$ & $0 \cdot 1473$ & 0.54 \\
\hline .853 & $0 \cdot 1237$ & $0 \cdot 1263$ & $0 \cdot 54$ \\
\hline 2878 & $0 \cdot 142 \pi$ & 1). 1073 & $0 \cdot 57$ \\
\hline- & $0 \cdot 1485$ & $0 \cdot 1015$ & $\ldots$ \\
\hline 3942 & $0 \cdot 1684$ & $v \cdot 0816$ & $0.5 \overline{5}$ \\
\hline 2900 & 0.1806 & 0.0694 & $0 \cdot 56$ \\
\hline 4395 & ). 1952 & 0.0548 & 0.54 \\
\hline \multirow[t]{2}{*}{5078} & $0 \cdot 2096$ & 0.0404 & 0.53 \\
\hline & & & 0.54 \\
\hline
\end{tabular}


Wie ersichtlich, mußten die Messungen gelegentlich unterbrochen und nach Auf hebung der Gassperre -- der Thermostat war mit Leuchtgas geheizt - wieder fortgesetzt werden. Die Konstanz von $k_{\text {ue }}$ ist eine ganz ausgezeichnete, woraus sich für die Verseifung das Konstantenverhältnis $2: 1$ ergibt. Eine Anfangsbeschleunigung isi entsprechend der geringen Stärke der Malonsäure und ihrer Estersäure nicht wahrzunehmen. Die Reaktion des Versuches 4, die weiter verfolgt wurde als die des Versuches 5, zeigt gegen Ende eine merkliche Hemmung, die auf die Gegenwirkung zurückzuführen ist. Bei Berechnung des Mittelwertes von $k_{a e}$ des Versuches 4 wurden daher die letzten Werte weggelassen.

Die Mittelwerte von $k_{a c}$ der Versuche 4 und 5 zeigen deutlich die Proportionalität der Verseifungsgeschwindiglseit mit der Konzentration der Katalysatorsäure, beziehungsweise der Wasserstoffionkonzentration.

\section{Die Verseifung von Diäthylmalonat.}

Die Untersuchungen wurden auf die gleiche Weise wie bei dem Malonsäuremethylester vorgenommen.

6. Versuch.

\begin{tabular}{|c|c|c|c|}
\hline$t_{y}-\cdots t_{1}$ & $T$ & $(a-x-y-y)_{d t^{\prime}}$ & $10+k_{a c}$ \\
\hline-- & $0 \cdot 1013$ & $0 \cdot 2682$ & $\ldots$ \\
\hline 272 & $0 \cdot 1085$ & 0.2610 & $1 \cdot 00$ \\
\hline 1195 & $0 \cdot 1379$ & $0 \cdot 2316$ & $1 \cdot 00$ \\
\hline 1359 & $0 \cdot 1677$ & 0.2018 & $1 \cdot 01$ \\
\hline 1754 & $0 \cdot 2011$ & $0 \cdot 1684$ & $1 \cdot 03$ \\
\hline 1104 & $0 \cdot 2196$ & $0 \cdot 1499$ & $1 \cdot 05$ \\
\hline 1458 & $0 \cdot 2409$ & $0 \cdot 1286$ & $1 \cdot 05$ \\
\hline 2923 & 0.2748 & $0 \cdot 0947$ & $1 \cdot 05$ \\
\hline- & $0 \cdot 3068$ & $0 \cdot 0628$ & - \\
\hline 2626 & 0.3207 & 0.0488 & $(0 \cdot 96)$ \\
\hline 2911 . & $0 \cdot 3321$ & 0.0374 & $(0.91)$ \\
\hline \multirow[t]{2}{*}{4268} & $0 \cdot 3429$ & $0 \cdot 0266$ & $(0 \cdot 80)$ \\
\hline & & & 1.03 \\
\hline
\end{tabular}


7. Versuch.

\begin{tabular}{|c|c|c|c|}
\hline$t_{y}-t_{1}$ & $\dot{T}$ & $(a-x-y) a c$ & $104 \mathrm{~kat}$ \\
\hline - & $0 \cdot 1016$ & $0 \cdot 1962$ & -- \\
\hline 1315 & $(9 \cdot 1259$ & $0 \cdot 1720$ & $1 \cdot 00$ \\
\hline - & $(1) \cdot 1460$ & 0.1518 & $-\ldots$. \\
\hline 2493 & $0 \cdot 180 \pi$ & $0 \cdot 1173$ & $1 \cdot u^{2}$ \\
\hline 1397 & $0 \cdot 196 t$ & $10 \cdot 1012$ & 1.06 \\
\hline 2940 & $0 \cdot 2242$ & 11.0736 & $1 \cdot 08$ \\
\hline \multirow[t]{2}{*}{2024} & $0 \cdot 2440$ & $0.0,338$ & $1 \cdot 07$ \\
\hline & & & $1 \cdot 05$ \\
\hline
\end{tabular}

Es sind dieselben Wahrnehmungen zu machen wie beim Malonsäuremethylester und daher sind auch die gleichen Schlußfolgerungen zu ziehen. Die Übereinstimmung des Mittels von $k_{a}$ bei beiden Versuchen beweist die gute Reproduzierbarkeit. Ein Vergleich der Vetsuche mit dem Versuch 4, dessen. Lösung gleich viel Katalysatorsäure enthält, lehrt, daß die Geschwindigkeit der Verseifung des Athylesters nicht merklich verschieden ist von der des Methylesters.

\section{Zusammenfassung.}

Bei der sauren Verseifung eines Dicarbonsäureesters zeigt bekanntlich der nach Äquivalenten berechnete Koeffizient erster Ordnung Konstanz, sowie das Verhältnis der Stufenverseifung $2: 1$ ist. Infolge von Nebenumständen ist die Konstanz des derart berechneten Koeffizienten beim Oxalsäureester nur eine beiläufige. Um auch bei diesem Ester das Konstantenverhältnis einwandfrei nachzuweisen, wurde neben dem Säuretiter auch der Neutralester, und zwar nach einer jodometrischen Methode bestimmt und aus beiden laufenden Konzentrationen das Konstantenverhältnis berechnet und $\mathrm{zu} 2: 1$ befunden.

Der Methylester der Oxalsäure verseift ungefähr doppelt so rasch als der Äthylester.

Ferner wurde gezeigt, daß auch für die saure Verseifung der Malonsäureester das Konstantenverhältnis $2: 1$ gilt und daß der Methylester und der Äthylester ungefähr gleich rasch verseifen, 